\title{
Weighted Correlation Coefficient with a Trigonometric Function Entropy of Intuitionistic Fuzzy Set in Decision Making
}

\author{
Wan Khadijah Wan Ismail, Lazim Abdullah* \\ School of Informatics and Applied Mathematics, Universiti Malaysia Terengganu, 21030 Kuala Terengganu, \\ Malaysia. \\ * Corresponding author. Tel.: 609 6683335; email: lazim_m@umt.edu.my \\ Manuscript submitted March 5, 2015; accepted June 9, 2015. \\ doi: 10.17706/ijapm.2015.5.3.199-205
}

\begin{abstract}
Weighted correlation coefficient is one of the methods for intuitionistic fuzzy sets based multi-criteria decision making (IFS-MCDM). It uses a method of entropy to find criteria weights for alternatives. Entropy weight that employed the arithmetic operations of addition and subtraction in IFS memberships is used for establishing the weighted correlation coefficient. However, the entropy obtained from the use of the arithmetic operations may deprive the significance of membership degree, non-membership degree and hesitation degree of IFS. This paper proposes a sine function entropy weight for measuring weighted correlation coefficients of IFS-MCDM. An example is given to illustrate the proposed method. The comparison results are also presented to show the feasibility and effectiveness of the proposed sine function entropy weight.
\end{abstract}

Key words: Entropy, correlation coefficient, intuitionistic fuzzy sets, sine function.

\section{Introduction}

Intuitionistic fuzzy set (IFS) was introduced by Atanassov [1] as the extension and generalization of Zadeh's fuzzy set [2]. The concept of dual memberships in IFS was well-received by many researchers thanks to the unique characteristics of memberships degree, non-membership degree and hesitation degrees. The IFS has successfully used in solving various applications of multi-criteria decision making (MCDM) problems. Past researchers have studied the applications of IFS in a diverse array of MCDM (IFS-MCDM) disciplines such as medical diagnostics [3], environment performance index [4], similarity measures [5], human capital [6] and pattern recognition [7]. In decision making process, sometimes the information about criteria weights is completely unknown due to lack of knowledge or data and limitations of experts in providing information about the problem domain [8], [9]. To address this issue, Ye [10] proposed an MCDM method based on weighted correlation coefficient using entropy weights. This weighted correlation coefficient measures the strength of correlation between an alternative and the ideal alternative in MCDM problem. Ye [10] used the entropy weight that was defined by Burillo \& Bustince [11] based on the knowledge of complementary membership degrees in IFS. The sum of membership degree, non-membership degree and hesitation degree must fulfill the unique maximum membership of 1 . It seems that the entropy proposed by Burillo \& Bustince [11] used a simple arithmetic operation. The arithmetic operation may deprive the importance of the three membership degrees, particularly in the situation where 
the degree of hesitation is small. In order to boost the significance of entropy weight in IFS-MCDM and to mine the importance of hesitation degree, a similar function with maximum value of 1 could be proposed. Parkash et al., [12] provide some clues over this matter by introducing weighted measures of fuzzy entropy based on the maximum entropy principle. However, the multiplication of weights to cyclic or trigonometric function in Parkash et al., [12] would exaggerate the contribution of weight to entropy. On the other hand, entropy is basically a measure of weight in perception based on theory of probability. As a result, Ye [10] proposed trigonometric function entropy specifically for IFS based on the fuzzy entropy of Parkash et al., [12]. The emergence of trigonometric or one unit cycle function of IFS entropy and the issue of maximum operations in complementary nature of IFS may shed some light on the improvement of weighted correlation coefficient in IFS-MCDM. Motivated by these works, the present paper proposes the weighted correlation coefficient using a trigonometric function of IFS entropy for solving IFS-MCDM. The proposed weighted correlation coefficient uses the sine function of IFS entropy instead of arithmetic operations of IFS entropy. In short, the objective of this paper is to introduce sine function based entropy weight of IFS in measuring weighted correlation coefficient.

\section{Preliminaries}

This section introduces the basic definitions relating to fuzzy set theory, IFS, intuitionistic fuzzy entropy and weighted correlation coefficient.

Out of several higher order fuzzy sets, IFS [1] is a primary extension of the conventional fuzzy sets theory. Both are alleviate some drawbacks of Zadeh's fuzzy set and have been found to be highly useful to deal with vagueness. The IFS allocates both membership and non-membership to each element of the universe.

Definition 1: Intuitionistic fuzzy sets [1]

An IFS in $X$ is an expression $A$ is defined by

$$
A=\left\{<x, \mu_{A}(x), v_{A}(x)>\mid x \hat{I} X\right\}
$$

where $\mu_{A}(x): X \rightarrow[0,1]$ and $v_{A}(x): X \rightarrow[0,1]$ with the condition $0 \leq \mu_{A}(x)+v_{A}(x) \leq 1$. The numbers $\mu_{A}(x)$ and $v_{A}(x)$ represent respectively the membership degree and non-membership degree of the element $x$ to the set $A$. For each IFS in $X$ :

$$
\pi_{A}(x)=1-\mu_{A}(x)-v_{A}(x)
$$

for all $x \in X$. Then $\pi_{A}(x)$ is called the intuitionistic index or hesitancy degree of the element $x$ in the set $A$. It can be seen that $0 \leq \pi_{A}(x) \leq 1, x \in X$.

Definition 2: Correlation coefficient of IFS [13]

Let $A$ and $B$ be two IFSs in the universe of discourse $X=\left\{x_{1}, x_{2}, \ldots, x_{n}\right\}$. The correlation coefficient of $A$ and $B$ is given by

$$
k(A, B)=\frac{C(A, B)}{\sqrt{T(A) \cdot T(B)}},
$$

where the correlation of two IFSs $A$ and $B$ is given by $C(A, B)=\sum_{i=1}^{n}\left(\mu_{A}\left(x_{i}\right) \mu_{B}\left(x_{i}\right)+v_{A}\left(x_{i}\right) v_{B}\left(x_{i}\right)\right)$ and 
the informational intuitionistic energies of two IFSs $A$ and $B$ are given by $T(A)=\sum_{i=1}^{n}\left(\mu_{A}^{2}\left(x_{i}\right)+v_{A}^{2}\left(x_{i}\right)\right)$ and $T(B)=\sum_{i=1}^{n}\left(\mu_{B}^{2}\left(x_{i}\right)+v_{B}^{2}\left(x_{i}\right)\right)$, respectively.

Definition 3: Weighted correlation coefficient [12].

The correlation coefficient between an alternative $A_{\mathrm{i}}$ and the ideal alternative $A^{*}$ with entropy weights for criteria defined as follows:

$$
W_{i}\left(A^{*}, A_{i}\right)=\frac{C_{i}\left(A^{*}, A_{i}\right)}{\sqrt{T^{*}\left(A^{*}\right) T_{i}\left(A_{i}\right)}}=\frac{\sum_{j=1}^{n} w_{j} \mu_{A}\left(C_{j}\right)}{\sqrt{\sum_{j=1}^{n} w_{j}\left(\mu_{A_{i}}^{2}\left(C_{j}\right)+v_{A_{i}}^{2}\left(C_{j}\right)\right)}} .
$$

when

$$
w_{j}=\frac{1-H_{j}}{n-\sum_{j=1}^{n} H_{j}}
$$

where $w_{j} \in[0,1], \sum_{j=1}^{n} w_{j}=1, H_{j}=\frac{1}{m} E\left(C_{j}\right)$ and $0 \leq H_{j} \leq 1$ for $(j=1,2, \ldots, n)$.

All the definitions provide the fundamental knowledge for decision making using weighted correlation coefficient.

\section{Weighted Correlation Coefficient with Sine Function Entropy}

Ye [10] developed weighted correlation coefficient with the aim to solve a decision problem. The method deals with the incomplete information where the criteria weights are unknown and the memberships of criteria may take in form of intuitionistic fuzzy sets. A new improvement of finding entropy is introduced in the proposed method. The basic entropy based on the definition of complementary in IFS is replaced with entropy based on trigonometric or cyclic function of sine. The maximum value of one in IFS is retained with the introduction of entropy weight sine function. The basic steps of the proposed weighted correlation coefficient are organized as follows.

Step 1: Construct a fuzzy decision matrix.

Evaluations of alternatives are represented by IFS. The intuitionistic fuzzy valued decision matrix $D=\left[d_{i j}\right]_{m}$ where $d_{i j}=\left(\mu_{i j}, v_{i j}\right)$ is the evaluation of alternative $A_{i}$ with respect to criteria $C_{j}$.

Step 2: Find the entropy weight of each criteria, $C_{j}$.

The trigonometric sine function of IFS entropy is proposed as

$$
E_{1}(A)=\frac{1}{n} \sum_{j=1}^{n}\left\{\frac{\sin \pi \times\left[1+\mu_{A}(x)-v_{A}(x)\right]}{4}+\frac{\sin \pi \times\left[1+\mu_{A}(x)+v_{A}(x)\right]}{4}\right\} \times \frac{1}{\sqrt{2}-1}
$$

Step 3: Find the weight of each criteria, $C_{j}$.

Since the weight of criteria is completely unknown, the weights of criteria can be obtained using entropy weight computed using Eq. (5).

Step 4: Find the weighted correlation coefficient. 
The correlation coefficient between an alternative $A_{i}$ and the ideal alternative $A^{*}$ with entropy weights for criteria is measured using Eq. (4).

Step 5: Rank the alternative $A_{i}(i=1,2,3, \ldots, m)$ and select the best one(s) in accordance with weighted correlation coefficient. The highest ranking or the best alternative is chosen based on the highest weighted correlation coefficient.

This paper proposes a modification to entropy weight, specifically in Step 2, without missing the generality of weighted correlation coefficient of Ye [10].

\section{Illustrative Example}

In order to demonstrate the feasibility of the weighted correlation coefficient, the authors consider the decision making problem discussed in [14].

Example. An air-condition system selection problem [14].

Suppose that there exist three air-conditions systems; $A_{1}, A_{2}$ and $A_{3}$ which form the set of all alternatives $A=\left\{A_{1}, A_{2}, A_{3}\right\}$. Suppose four criteria $C_{1}$ (good quality), $C_{2}$ (easy to operate), $C_{3}$ (economical) and $C_{4}$ (good service after selling) are taken into account in this problem. Now it is a need to choose an air-condition system which satisfies $C_{1}, C_{2}, C_{3}$ and $C_{4}$.

The selection process is executed using the proposed method.

Step 1: Construct a fuzzy decision matrix.

$$
\begin{aligned}
& \begin{array}{ccccc}
C_{1} & C_{2} & C_{3} & C_{4}
\end{array} \\
& D=\begin{array}{l}
A_{1} \\
A_{2} \\
A_{3}
\end{array}\left[\begin{array}{cccc}
(0.85,0.10) & (0.75,0.10) & (0.70,0.15) & (0.70,0.10) \\
0.70,0.10) & (0.80,0.15) & (0.85,0.10) & (0.70,0.15) \\
(0.80,0.10) & (0.80,0.10) & (0.75,0.15) & (0.70,0.15)
\end{array}\right]
\end{aligned}
$$

Step 2: Find the entropy of each criteria, $C_{j},(j=1,2,3,4)$ using Eq. (6).

$$
E\left(C_{1}\right)=\frac{1}{3}\left[\left[\left\{\frac{\sin \pi \times[1+0.85-0.10]}{4}+\frac{\sin \pi \times[1+0.85+0.10]}{4}\right\} \times \frac{1}{\sqrt{2}-1}\right]+\ldots+\ldots\right]=4.7397
$$

The entropy for other criteria $E\left(C_{2}\right), E\left(C_{3}\right)$ and $E\left(C_{4}\right)$ are computed with the similar fashion. It is obtained as $E\left(C_{2}\right)=4.7470, E\left(C_{3}\right)=4.7166$ and $E\left(C_{4}\right)=4.6703$.

Step 3: Find the weights of each criteria, $C_{j},(j=1,2,3,4)$ using Eq. (5).

$$
w_{1}=\frac{|1-1.5799|}{|4-6.2912|}=0.2531 \text { where } H_{1}=\frac{1}{3}(4.7397)=1.5799 \text { and } \sum_{j=1}^{4} H_{j}=6.2912
$$

With the similar calculation, weights for the rest of criteria are $w_{2}=0.2542, w_{3}=0.2497$ and $w_{4}=0.2430$. 
Step 4: Find the weighted correlation coefficient.

The correlation coefficient between an alternative $A_{i}$ and the ideal alternative $A^{*}$ with entropy weights for criteria is measured using Eq. (4).

For the alternative $1, A_{1}$, the weighted correlation coefficient is given as

$$
W_{1}\left(A^{*}, A_{1}\right)=\frac{[0.2531(0.85)]+\ldots+\ldots+[0.2430(0.70)]}{\sqrt{\left[0.2531\left(0.85^{2}+0.10^{2}\right)\right]+\ldots+\ldots+\left[0.2430\left(0.70^{2}+0.10^{2}\right)\right]}}=0.9853
$$

With the similar fashion, the weighted correlation coefficients for other alternatives are $W_{2}\left(A^{*}, A_{2}\right)=0.9829$ and $W_{3}\left(A^{*}, A_{3}\right)=0.9843$.

Step 5: Rank the alternative $A_{i}(i=1,2,3)$ according the value of weighted correlation coefficient. The highest weight is the best alternative.

$A_{1}>A_{3}>A_{2}$.

Therefore, the air-condition system $\mathrm{A}_{1}$ is chosen as an ideal air-condition system which satisfies $C_{1}, C_{2}$, $C_{3}$ and $C_{4}$. Thus, the ranking result is consistent with the result obtained by Liu \& Wang [14]. The problem of Liu \& Wang [14] was tested using the score function. The same problem was also tested by Wu \& Zhang [15] using intuitionistic fuzzy weighted entropy. The results from these two tests were consistent with the result using the proposed method. It is also worth to note here that Ye [10] used the complementary arithmetic operations of IFS in finding entropy weight. The comparative results of the problem with different approaches are shown in Table 1.

Table 1. Ideal Air-Condition System: A Comparative Result

\begin{tabular}{ll}
\hline Method & The selected air-condition system \\
\hline Score function [10] & $\mathrm{A}_{1}$ \\
Weighted correlation coefficient with arithmetic & $\mathrm{A}_{1}$ \\
$\begin{array}{l}\text { operations [14] } \\
\text { Intuitionistic fuzzy weighted entropy [15] }\end{array}$ & $\mathrm{A}_{1}$ \\
$\begin{array}{l}\text { The proposed weighted correlation coefficient with } \\
\text { Sine function }\end{array}$ & $\mathrm{A}_{1}$ \\
\hline
\end{tabular}

The result shows that the alternative $A_{1}$ is consistently selected as an ideal air-condition system albeit different methods used.

\section{Conclusion}

The paper presented a new approach for determining the entropy weight in evaluating the weighted correlation coefficient. To determine the criteria weights, a sine function entropy weight proposed by Ye [10] is used to replace the entropy proposed by Burillo \& Bustince [11] in which the latter used the arithmetic operation in the relationship of IFS memberships in proposing entropy. The entropy weights were adapted into the weighted correlation coefficient. The alternatives could be ranked accordingly and the most desirable one(s) can be selected according to the weighted correlation coefficient. Finally, an illustrative example was implemented to demonstrate the feasibility of the proposed method. Validation with the other methods was also provided as a comparative analysis.

\section{Acknowledgment}

This work is part of the project FRGS 59243. Thanks are due to Malaysian Ministry of Education and 
Universiti Malaysia Terengganu.

\section{References}

[1] Atanassov, K. (1986). Intuitionistic fuzzy sets. Fuzzy Sets and Systems, 20(1), 87-96.

[2] Zadeh, L. A. (1965). Fuzzy sets. Information and Control, 8(3), 338-353.

[3] De, S. K., Biswas, R., \& Roy, A. R. (2001). An application of intuitionistic fuzzy set in medical diagnosis. Fuzzy Sets Systems, 117(2), 209-213.

[4] Abdullah, L., \& Wan, I. W. K. (2013). A new ranking of environmental performance index using weighted correlation coefficient in intuitionistic fuzzy sets: A case of ASEAN countries. Modern Applied Science, $7(6), 42-52$.

[5] Yusoff, B., Taib, I., Abdullah, L., \& Wahab, A. F. (2011). A new similarity measure on intuitionistic fuzzy sets. International Journal of Computational and Mathematical Sciences, 5(2), 70-74.

[6] Abdullah, L., Jaafar S., \& Taib, I. (2013). Intuitionistic fuzzy analytic hierarchy process approach inranking of human capital indicators. Journal of Applied Sciences, 13(3), 423-429.

[7] Dengfeng, L., \& Chuntian, C. (2002). New similarity measure of intuitionistic fuzzy sets and application to pattern recognitions. Pattern Recognition Letter, 23(1-3), 221-225.

[8] Chou, S. Y., Chang Y. H., \& Shen, C. Y. (2008). A fuzzy simple additive weighting system under group decision-making for facility location selection with objective/subjective attributes. European Journal of Operation Research, 189(1), 132-145.

[9] Yeh, C. H., \& Chang, Y. H. (2009). Modelling subjective evaluation for fuzzy group multi-criteria decision making. European Journal of Operation Research, 179, 220-233.

[10] Ye, J. (2010). Fuzzy decision-making method based on the weighted correlation coefficient under intuitionistic fuzzy environment. European Journal of Operation Research, 205, 202-204.

[11] Burillo, P., \& Bustince, H. (1996). Entropy on intuitionistic fuzzy sets and on interval-valued fuzzysets. Fuzzy Sets and Systems, 19, 305-316.

[12] Parkash, O., Sharma P. K., \& Mahajan, R. (2008). New measures of weighted fuzzy entropy and their applications for the study of maximum weighted fuzzy entropy principle. Information Sciences, 178(11), 2389-2395.

[13] Gernsternkorn, T., \& Manko, J. (1991). Correlation of intuitionistic fuzzy sets. Fuzzy Sets and Systems, 44, $39-43$.

[14] Liu, H. W., \& Wang, G. J. (2007). Multi-criteria decision-making methods based on intuitionistic fuzzy sets. European Journal of Operation Research, 179, 220-233.

[15] Wu, J. Z., \& Zhang, Q. (2011). Multi-criteria decision making method based on intuitionistic fuzzy weighted entropy. Expert Systems with Applications, 38, 916-922.

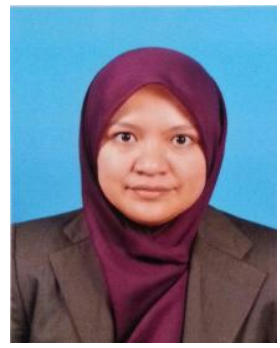

Wan Khadijah Wan Ismail is a research student at the School of Informatics and Applied Mathematics, Universiti Malaysia Terengganu. Her research interests include fuzzy sets and similarity measures. 


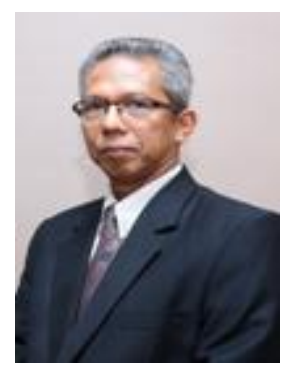

Lazim Abdullah is a professor at the School of Informatics and Applied Mathematics, Universiti Malaysia Terengganu. He holds a B.Sc (Hons) in mathematics from the University of Malaya, Kuala Lumpur in June 1984 and the M.Ed in mathematics education from University Sains Malaysia, Penang in 1999. He received his Ph.D. from the Universiti Malaysia Terengganu, (information technology development) in 2004. His research focuses on the mathematical theory of fuzzy sets and its applications in social ecology, environmental sciences, health sciences, and manufacturing engineering. He is interested in the measurement of social indicators, indices of health related quality of life and environmental index using computational intelligence and statistical approaches. His research findings have been published in over two hundred publications including refereed journals, conference proceedings, and chapters in book, and research books. Currently he is a member of editorial boards to several international journals related with computing and information technology. Besides, he is also a reviewer for number of local and international journals, member of scientific committees of several symposia and conferences at national and international levels. Dr. Lazim is an associate member of IEEE Computational Intelligence Society, a member of Malaysian Mathematical Society and a member of the International Society on Multiple Criteria Decision Making. 